\title{
UM ALGORITMO POLINOMIAL PARA O PROBLEMA DE EMPACOTAMENTO DE CONTÊINERES COM ESTABILIDADE ESTÁTICA DA CARGA
}

\author{
José Lassance de Castro Silva \\ Nei Yoshihiro Soma* \\ Instituto Tecnológico de Aeronáutica \\ São José dos Campos - SP \\ lassance@,comp.ita.cta.br \\ nysoma@comp.ita.cta.br \\ * Corresponding author/autor para quem as correspondências devem ser encaminhadas \\ Recebido em 03/2002, aceito em 11/2002 após 1 revisão
}

\begin{abstract}
Resumo
O objetivo deste artigo é apresentar um algoritmo polinomial na quantidade de recursos computacionais utilizados, que encontre boas soluções para o problema de empacotamento de bins tridimensionais; tendo como maior contribuição, considerações sobre a estabilidade estática da carga. Os critérios para o empacotamento estável dos itens dentro dos bins são apresentados com detalhes. O procedimento para o preenchimento dos itens dentro dos bins utiliza o bem conhecido princípio da alocação em pontos de cantos. O problema é NP-difícil no sentido forte e extremamente difícil de ser resolvido na prática. Apresenta-se grande quantidade de experimentos computacionais para problemas com até 90 itens (com e sem estabilidade estática) e os resultados são comparados com aqueles obtidos da literatura.
\end{abstract}

Palavras-chave: bin packing tridimensional; empacotamento com estabilidade estática.

\begin{abstract}
A polynomial computational bounded resources heuristic for the Three-Dimensional Bin Packing Problem (3D-BPP) is suggested. The major contribution is the introduction of static stability of the packing and the criteria used for such a packing are described in details. The filling process follows the well known 3-D corner approach. The problem is NP-Hard in the strong sense and extremely difficult to be solved in the practice. Extensive computational experiments are also presented, with instances up to 90 items, with and without static stability. The results are compared with those obtained from the literature.
\end{abstract}

Keywords: three-dimensional bin packing problem; packing with static stability. 


\section{Introdução}

Problemas de corte e empacotamento têm sido extensivamente tratados na literatura. Devido à diversidade de casos em que esses problemas podem aparecer na prática, e a complexidade estrutural dos mesmos (os problemas são em geral NP-difíceis), faz com que a grande maioria dos trabalhos encontrados na literatura apresente abordagens heurísticas para a resolução desses. O principal objetivo deste artigo é o de apresentar uma técnica que encontre boas soluções para o problema de empacotamento de bins tridimensionais $(3 D-B P P)$ com a restrição adicional que os itens empacotados estejam em equilibrio dentro dos bins. Note-se que no corpo do trabalho, os termos bin tridimensional e container são tratados de maneira não distinta.

O 3D-BPP tem como dados de entrada:

- um conjunto de $n$ itens (caixas retangulares), cada um desses itens sendo caracterizados pela largura $\mathrm{wj}$, altura hj e comprimento dj, referenciado por uma tripla ordenada $\left\{w_{j}, h_{j}, d_{j}\right\}$, ou seja, e.g. $\left\{w_{j}, h_{j}, d_{j}\right\} \neq\left\{h_{j}, w_{j}, d_{j}\right\}$ (rotações implicam em itens distintos), para todo $j \in J=\{1,2, \ldots, \mathrm{n}\}$;

- os itens não necessitam serem feitos de um mesmo material, muito embora, supõe-se que todos esses possuam uma mesma densidade, são perfeitamente rígidos, além de serem homogêneos; e

- um número limitado de bins (contêineres ou caixas retangulares maiores), onde inicialmente há uma disponibilidade de pelo menos $n$ bins idênticos e tridimensionais, tendo dimensões, internas, comuns: largura $W$, altura $H$ e comprimento $D$.

Também, supõe-se, sem perda de generalidade, que todos os dados de entrada são números inteiros positivos, satisfazendo às relações: $w_{j} \leq W, h_{j} \leq H$ e $d_{j} \leq D$, para todo $j \in J$.

A resolução do 3D-BPP consiste em se empacotar ortogonalmente todos os $n$ itens, utilizando a menor quantidade possível de bins. É importante notar, uma vez mais, que na variante do problema aqui abordado, exige-se que o empacotamento dos itens seja estável e que no melhor do conhecimento dos autores, não há menção a isto na literatura.

As motivações práticas para o estudo do 3D-BPP vêm da grande quantidade de aplicações industriais por ele modelados, as quais variam do carregamento de cargas em aviões ao seqüenciamento (escalonamento) de tarefas em ambientes multi-processados. Sob o ponto de vista teórico, o problema é uma extensão natural do bem conhecido e já clássico problema Bin Packing (BPP), cf. Garey \& Johnson (1979) sendo ele também, NP-difícil no sentido forte.

Ressalve-se que, contrariamente ao Bin Packing usual (unidimensional), não se conhece heurística alguma, s.m.j., para o 3D-BPP com garantia de desempenho, e. g. para o BPP, a bem conhecida heurística FFD implica em um erro assintótico de 11/9 e um tempo de processamento limitado por $O(\mathrm{n} \cdot \log \mathrm{n})$.

Verifica-se que o 3D-BPP começa a ser pesquisado, mais intensivamente, há pouco mais de dez anos. Conforme especificado em Dowsland (1991), há quantidade restrita de trabalhos publicados relatando tais problemas de empacotamentos tridimensionais. A primeira heurística apresentada para o 3D-BPP aparece em Scheithauer (1991).

Chen et al. (1995) desenvolveram um modelo analítico para o problema do carregamento de contêineres $(P C C)$, no qual se inclui o 3D-BPP de forma indireta. O modelo consiste em 
selecionar um número de bins para empacotar um conjunto dado de itens. Os bins não precisam ser idênticos e os itens não necessitam serem colocados ortogonalmente. O objetivo principal daquela abordagem é o de se minimizar o espaço desocupado dentro dos bins, onde alguns casos especiais do problema também são lá discutidos. O modelo exato é de programação linear inteira mista zero-um, onde mesmo para problemas pequenos a resolução prática dos mesmos torna-se computacionalmente inviável, tanto em termos de tempo de execução quanto em memória.

A formulação matemática para o 3D-BPP, sem levar em conta as condições de estabilidade estática dos itens dentro dos bins, pôde ser obtida a partir do modelo de Chen et al. (1995), para o PCC, através de duas modificações no mesmo que são:

- A substituição da função objetivo do modelo do PCC, que minimiza o espaço não utilizado nos bins selecionados para o empacotamento de um conjunto de itens, por minimizar o número de bins utilizados para empacotar todos os itens dados;

- Atribuição de valores específicos um grupo de variáveis do PCC $\left(w_{x i}=l_{z i}=h_{y i}=1 \mathrm{e}\right.$ $w_{y i}=w_{z i}=l_{x i}=l_{y i}=h_{x i}=h_{z i}=0$ ) para evitar que rotações nos itens sejam permitidas.

Desta forma o 3D-BPP tem a seguinte formulação matemática:

$$
\operatorname{Min} \sum_{i=1}^{n} \eta_{i}
$$

Sujeito à:

$$
\begin{aligned}
& x_{j}+w_{j} \leq x_{k}+\left(1-a_{j k}\right) M, \forall j, k \in J e j<k \\
& x_{k}+w_{k} \leq x_{j}+\left(1-b_{j k}\right) M, \forall j, k \in J e j<k \\
& y_{j}+h_{j} \leq y_{k}+\left(1-e_{j k}\right) M, \forall j, k \in J e j<k \\
& y_{k}+h_{k} \leq y_{j}+\left(1-f_{j k}\right) M, \forall j, k \in J e j<k \\
& z_{j}+d_{j} \leq z_{k}+\left(1-c_{j k}\right) M, \forall j, k \in J e j<k \\
& z_{k}+d_{k} \leq z_{j}+\left(1-g_{j k}\right) M, \forall j, k \in J e j<k \\
& a_{j k}+b_{j k}+c_{j k}+e_{j k}+f_{j k}+g_{j k} \geq \alpha_{j i}+\alpha_{k i}, \forall j, k \in J, j<k e 1 \leq i \leq m \\
& \sum_{i=1}^{m} \alpha_{j i}=1, \forall j \in J \\
& \sum_{j=1}^{n} \alpha_{j i} \leq M \eta_{i}, \forall 1 \leq i \leq m \\
& x_{j}+w_{j} \leq W+\left(1-\alpha_{j i}\right) M, \forall j \in J e 1 \leq i \leq m \\
& y_{j}+h_{j} \leq H+\left(1-\alpha_{j i}\right) M, \forall j \in J e 1 \leq i \leq m \\
& z_{j}+d_{j} \leq D+\left(1-\alpha_{j i}\right) M, \forall j \in J e 1 \leq i \leq m \\
& a_{j k}, b_{j k}, c_{j k}, e_{j k}, f_{j k}, g_{j k}, \alpha_{j i} e \eta_{i} \in\{0,1\}, \forall j, k \in J, j<k e 1 \leq i \leq m \\
& \quad x_{j}, y_{j}, z_{j} \geq 0, \forall j \in J
\end{aligned}
$$


onde:

$n$ é a quantidade de itens;

$m$ é o número de bins disponíveis, onde se supõe, sem perda de generalidade que $m=n$;

$M$ é um número arbitrário bastante grande;

$\alpha_{\mathrm{ji}}$ é uma variável binária que é igual a 1 se o item $j$ é colocado no bin $i$, caso contrário ela é igual a 0 ;

$\eta_{\mathrm{i}}$ é uma variável binária que é igual a 1 se o bin $i$ é usado, caso contrário ela é igual a 0 ;

$\left(x_{j}, y_{j}, z_{j}\right)$ variáveis contínuas (para localização dos itens) tendo como origem as coordenadas do canto inferior esquerdo do item $j$;

$a_{j k}, b_{j k}, c_{j k}, e_{j k}, f_{j k}, g_{j k}$ são as variáveis binárias definidas para indicar a colocação do item $j$ em relação ao item $k$.

A variável $a_{j k}$ é igual a 1 se o item $j$ é colocado do lado esquerdo do item $k$. Similarmente, as variáveis $b_{j k}, c_{j k}, e_{j k}, f_{j k}$ e $g_{j k}$ representam quando o item $j$ está à direita, atrás, abaixo, acima ou à frente do item $k$, respectivamente. Estas variáveis são necessárias e definidas somente quando $j<k$. Sem perda de generalidade, supõe-se que cada contêiner é colocado com a sua largura ao longo do eixo $X$, sua altura ao longo do eixo $Y$, enquanto que seu comprimento fica ao longo do eixo $Z$, do sistema de coordenadas espaciais, com a origem sendo definida no canto inferior esquerdo mais ao fundo do bin ou contêiner.

A resolução do modelo fornece uma solução ótima para o 3D-BPP. A função objetivo deste modelo implica em minimizar o número de bins utilizados para empacotar todos os $n$ itens dados no problema. As restrições (I.2) à (I.7) asseguram que os itens não vão ocupar o mesmo espaço dentro de um mesmo bin. A restrição (I.8) verifica se há sobreposição de itens, e isto é realizado somente para itens que estão colocados no mesmo bin. A garantia de que cada item será colocado em exatamente um bin está descrita na restrição (I.9). Um bin é considerado utilizado quando algum item é a ele alocado, este requisito é descrito na restrição (I.10). As restrições (I.11) à (I.13) asseguram que todos os itens colocados dentro de um bin encaixam-se dentro de suas dimensões físicas.

A resolução do problema utilizando a este modelo pode ser feita através do uso de pacotes comerciais de programação matemática tais como LINDO, CPLEX e etc. Todavia, a quantidade de restrições $\left(O\left(\mathrm{n}^{3}\right)\right)$ e variáveis $\left(O\left(\mathrm{n}^{2}\right)\right)$ tornam inviável sua resolução prática.

Em termos heurísticos, Pisinger et al. (1999) adaptaram para o 3D-BPP uma nova metodologia de aproximação para problemas da otimização combinatória, denominada Busca Local Direcionada GLS (Guided Local Search). Das meta-heurísticas bastante utilizadas para resolver problemas de otimização, e.g. Busca Tabu, Algoritmos Genéticos (AG) e etc., só há uma aplicação de AG, Corcoran \& Wainwright (1992), mas resolvendo o caso de preenchimento de itens por intermédio de faixas bi-dimensionais.

Em Martello et al. (2000) tem-se o primeiro algoritmo exato para o problema (3D-BPP), assim como para o do Carregamento de Contêineres (Container Loading Problem), para os quais uma abordagem branch-and-bound foi utilizada. Naquele mesmo artigo, duas heurísticas são propostas $\left(\mathrm{H}_{1}\right.$ e $\left.\mathrm{H}_{2}\right)$, as quais serão comentadas com mais detalhes adiante, já que uma delas $\left(\mathrm{H}_{1}\right)$ é base de comparação para a heurística aqui sugerida. Em todos esses artigos não há menção alguma à estabilidade dos itens empacotados dentro dos bins. 
$\mathrm{O}$ ataque ao 3D-BPP sugerido aqui se baseia em uma abordagem heurística a qual apresenta bons resultados práticos. Duas versões para a heurística são propostas, na primeira a estabilidade do empacotamento nos bins não é considerada, enquanto na segunda versão sim, sendo que as duas versões são comparadas com a melhor heurística conhecida da literatura.

A organização do trabalho segue: na Seção 2, são apresentadas as idéias básicas da heurística polinomial. Na Seção 3, os critérios para que o empacotamento dos itens dentro dos bins seja estável e a incorporação desses à heurística são descritos; enquanto que na Seção 4 são apresentados os resultados obtidos nos experimentos computacionais. As conclusões estão na Seção 5 .

\section{A Heurística Proposta: primeira versão}

A primeira versão da heurística para resolver o 3D-BPP, na qual a estabilidade estática não é considerada, será chamada de Heurística Sem Estabilidade Estática (HSEE). Por vício de linguagem a palavra versão é omitida, idem para o caso com estabilidade; reitera-se que a heurística é uma única, mas com duas versões.

HSEE tem como idéia básica, a política do melhor preenchimento possível de um único bin. Tal abordagem pode ser considerada como sendo "on line" nos bins e não nos itens como usual; para o BPP o melhor preenchimento (e conseqüente "fechamento") de um único bin apresenta bons resultados práticos, $c f$. Soma et al. (1999) tendo sido utilizada, também, no 3D-BPP em Martello et al. (2000).

Para o preenchimento de um bin é necessário que haja uma política que determine a ocupação do espaço interno dos bins. Neste sentido, assume-se que uma dada disposição espacial formada por um conjunto de itens já empacotados $(E)$ no bin, definirá duas regiões no mesmo: uma região chamada de "envelope", associado ao empacotamento daqueles itens de $E$, e outra região "vazia", na qual os itens ainda não alocados $(\Phi)$ podem, eventualmente, ser fisicamente alocados naquele bin.

Tanto a região "vazia" dentro de um bin, quanto seu complemento, podem ser determinados como funções de alguns pontos extremos nos quais itens podem ser inseridos, 3D-Corner, (ponto de canto); sendo esta definição, uma extensão do caso bi-dimensional, $c f$. Martello \& Vigo (1998). Um ponto de canto (PC) de um dado bin, seja ele $p$, indica que nenhum item $j \in \Phi$ poderá ter alguma interseção à direita de $p$, ou acima de $p$, ou defronte a $p$, em relação ao conjunto $E$ no bin em questão, caso $j$ houvesse que ser inserido em $p$. A representação de $p$ é feita usando-se o sistema de coordenadas cartesianas $\left(x_{p}, y_{p}, z_{p}\right)$, com a origem sendo definida no canto inferior esquerdo mais ao fundo do bin. Note-se que caso um item $j \in \Phi$ não possa ser alocado físicamente em $p$, pelo menos uma das condições, $x_{p}+w_{j} \leq W$ ou $y_{p}+h_{j} \leq H$, ou $z_{p}+d_{j} \leq D$ deve ser violada.

Na Figura 1 tem-se um conjunto de itens já alocados e os respectivos pontos de cantos (círculos pretos maiores). No processo de determinação dos PC's alguns pontos de cantos "falsos" (pontos brancos, Figura 1) podem ser gerados, os quais são facilmente detectados e removidos utilizando-se a dominância de pontos. Por exemplo, os pontos $a$ e $b$, representados na mesma figura e, respectivamente, com coordenadas $\left(x_{a}, y_{a}, z_{a}\right)$ e $\left(x_{b}, y_{b}, z_{b}\right)$ mostram que somente $a$ é um PC, pois $a$ está "escondido" atrás de $b$. Note-se que $x_{a}=x_{b}$, $y_{a}=y_{b}, z_{b}>z_{a}$ (critério de dominância). 


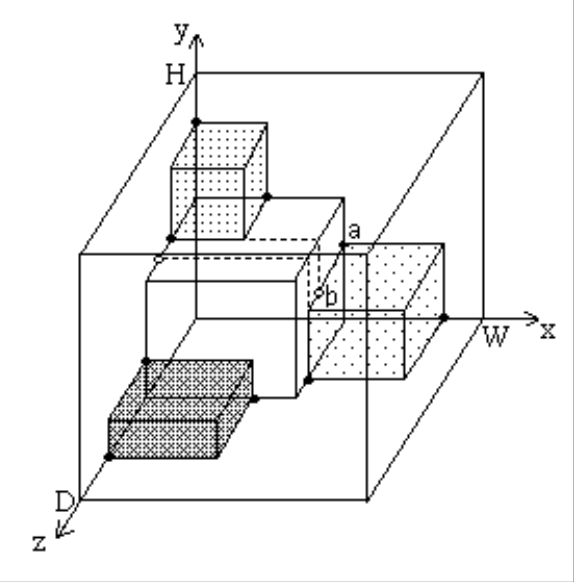

Figura 1 - Itens já empacotados com seus PC's (círculos negros maiores).

A heurística HSEE trabalha basicamente com duas listas ordenadas: $(\Phi)$ uma lista de itens que ainda tem que ser empacotados e a corrente lista de pontos de cantos $(\Lambda)$, sendo que a idéia básica é a de se alocar o primeiro item disponível em $\Phi$ ao primeiro ponto de canto fisicamente viável de $\Lambda$. Após a alocação, há uma atualização em $\Phi$, e assim por diante, até que $\Phi=\varnothing$. A lista $\Phi$ é ordenada obedecendo a cinco regras distintas (associada à variável inteira $O I$, com $1 \leq O I \leq 5$ ). Ainda, a lista $\Lambda$, também, é ordenada por sete regras distintas (dada pela variável inteira $O C P$, com $1 \leq O C P \leq 7$ ).

A lista de itens $\Phi$ ordenada conforme $O I$ pode ser vista como uma solução viável para o 3D-BPP. A seguir descrevemos as regras para cada um dos valores de $O I$ :

$$
O I=\left\{\begin{array}{l}
1, \text { os itens são classificados em ordem não - crescente do volume } \\
2, \text { os itens são classificados em ordem não - crescente do } \max \left\{w_{j}, h_{j}, d_{j}\right\} \\
3, \text { os itens são classificados em ordem não - crescente da largura } w_{j} \\
4, \text { os itens são classificados em ordem não - crescente da altura } h_{j} \\
5, \text { os itens são classificados em ordem não - crescente do comprimento } d_{j}
\end{array}\right.
$$

A lista dos pontos de cantos é definida por um conjunto de sete regras $(O C P)$, onde cada um dos valores de $O C P$ induz uma ordem parcial na correspondente lista de pontos de cantos, dado por:

$$
O C P=\left\{\begin{array}{l}
\text { 1, os PC são classificados em ordem não - decrescente da distância à origem } \\
\text { 2, os PC são classificados em ordem não - crescente da coordenada } x \\
3, \text { os PC são classificados em ordem não - decrescente da coordenada } x \\
4, \text { os PC são classificados em ordem não - crescente da coordenada } y \\
5, \text { os PC são classificados em ordem não - decrescente da coordenada } y \\
\text { 6, os PC são classificados em ordem não - crescente da coordenada } z \\
\text { 7, os PC são classificados em ordem não - decrescente da coordenada } z
\end{array}\right.
$$


O valor da variável $O C P$ determina a forma de preenchimento dos bins pelos itens. A Figura 2 mostra duas formas de preencher um dado bin, uma onde $O C P=1$ e uma outra onde $O C P=6$, para o mesmo conjunto de itens ordenados por $O I=4$.

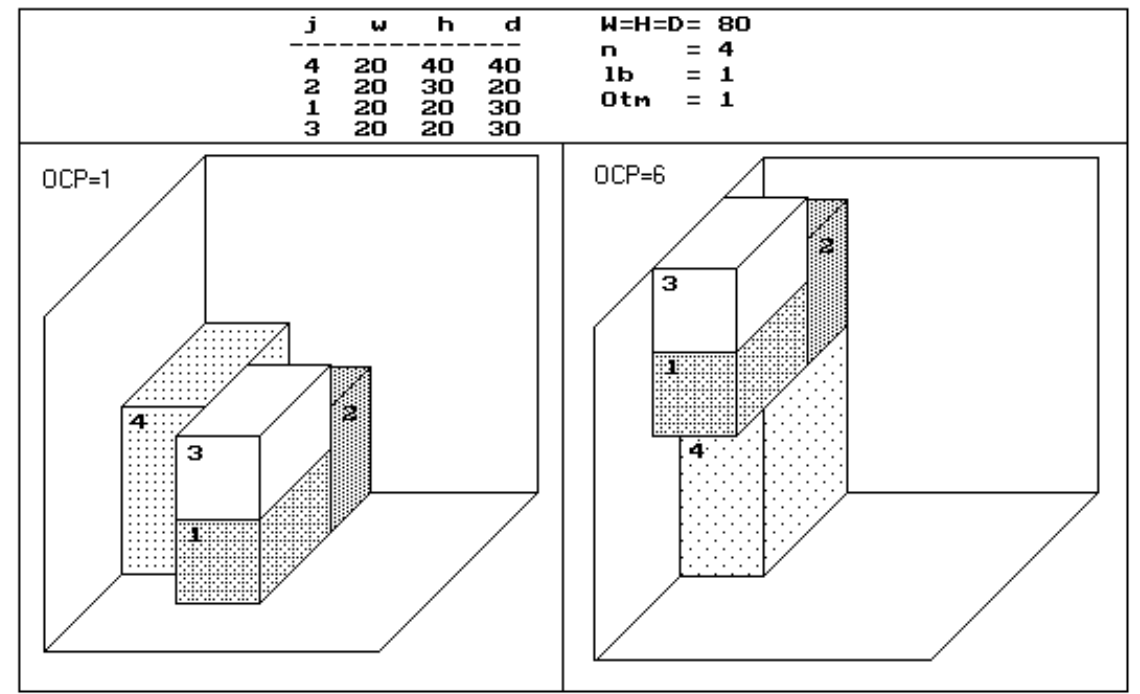

Figura 2 - Preenchimento do bin dado pela variável $O C P=1$ e $O C P=6$.

A heurística HSEE determina no máximo 35 soluções viáveis para o problema e como solução do problema o menor valor dentre essas soluções viáveis é escolhido. Há ainda, um critério de parada adicional, quando a solução ótima do problema é encontrada; para tanto, utiliza-se um limitante inferior, $\mathrm{L}_{2}$, onde as idéias gerais de seu desenvolvimento $\left(\mathrm{L}_{0} \rightarrow \mathrm{L}_{1} \rightarrow \mathrm{L}_{2}\right)$ são sumarizadas a seguir:

- O limitante inferior contínuo $\mathrm{L}_{0}$ : em que as dimensões dos itens não precisam ser inteiros positivos, i. e., uma fração de um item pode ser empacotada dentro de um bin;

- O limitante inferior $\mathrm{L}_{1}$ : obtido por redução ao BPP usual, onde não se leva em consideração no empacotamento, os itens, como sendo tridimensionais;

- O limitante inferior $\mathrm{L}_{2}$ : análogo ao limitante inferior $\mathrm{L}_{1}$, entretanto todas as três dimensões dos itens são consideradas. Em todos os experimentos aqui apresentados o limitante inferior $\mathrm{L}_{2}$ é utilizado, por ele dominar tanto $\mathrm{L}_{0}$ quanto $\mathrm{L}_{1}, c f$. Martello et al. (2000). Notando ainda, que $\mathrm{L}_{2}$ é determinado em tempo quadrático, ref. op. cit., na quantidade de itens.

Na Figura 3 a seguir tem-se a estrutura geral da heurística. 


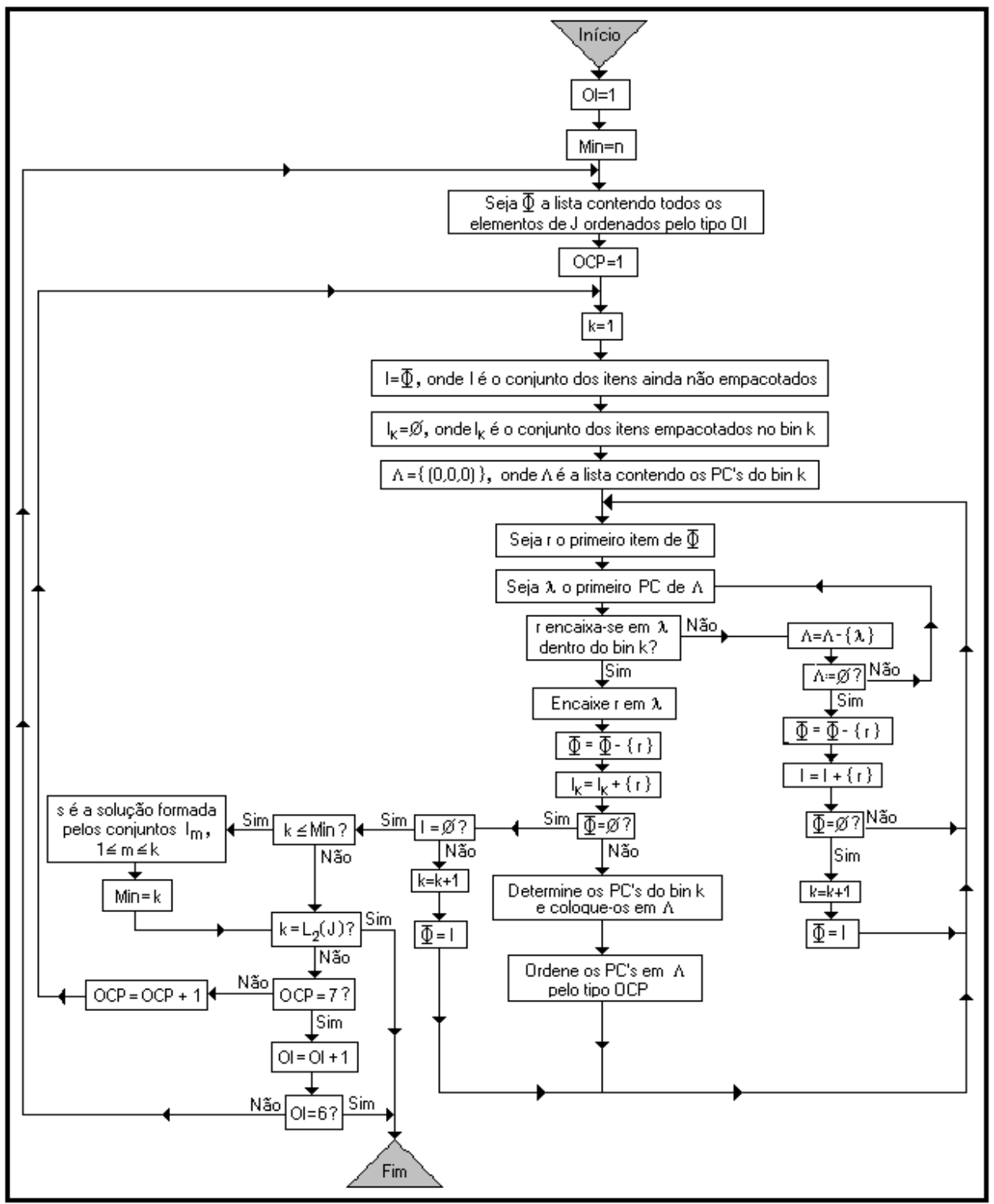

Figura 3 - Estrutura da Heurística Cúbica.

Para o cálculo da complexidade de HSEE, deve-se notar primeiramente que a quantidade máxima de PC's é linear em $n$, e identificada recursivamente por:

$\mathrm{T}_{0}=1$;

$\mathrm{T}_{1}=\mathrm{T}_{0}+2=1+2=3$;

$\mathrm{T}_{2} \leq \mathrm{T}_{1}+2=3+2=5$;

$\mathrm{T}_{\mathrm{n}} \leq \mathrm{T}_{\mathrm{n}-1}+2$, ou ainda $\mathrm{T}_{\mathrm{n}} \leq 2 n+1$. Isto implica que $\mathrm{T}_{\mathrm{n}}=O(n)$, para $n>0$. 
HSEE é limitada no tempo de processamento por $O\left(5 n \log n+n O\left(n^{2}\right)+7 n O(n) O(n \log n)\right)$; onde, a primeira parcela da soma determina o número de vezes que o conjunto de itens é ordenado, a segunda parcela está associada ao cálculo de $\mathrm{L}_{2}$ e na última, o laço principal de HSEE que usa 5 formas de classificar o conjunto de itens, com $n$ itens a serem empacotados, e caso todos eles pudessem ser alocados, ao máximo de $(2 n+1)=O(n)$ pontos de cantos. Ainda, que os PC's são ordenados de 7 formas distintas, conforme definição de $O C P$, lembrando que a ordenação de $n$ itens é realizada em $O(n \log n) c f$. Cormen et al. (1990). Logo, HSEE tem como limite de tempo de processamento $O\left(n^{3} \log n\right)$.

\section{Estabilidade Estática no Empacotamento}

Nesta Seção é mostrado como tratar a estabilidade estática dos itens (caixas) no empacotamento dentro dos bins, sendo que os estudos estão baseados em Beer \& Johnston (1976) e Meriam \& Kraige (1986), que tratam da mecânica dos corpos rígidos. Os conceitos utilizados para a determinação do equilíbrio estático de corpos rígidos (itens) baseiam-se nos conceitos bastante conhecidos de centro de gravidade, resultante de forças e de momentos, mas que serão aqui re-apresentados no contexto do problema.

Em um item, seja ele $j$, que foi suposto ser um paralelepípedo rígido e homogêneo, com seu peso uniformemente distribuído, o centro de gravidade $\mathrm{G}$ irá se localizar na metade de cada uma das suas dimensões. Se $j$ tiver dimensões $\mathrm{w}_{\mathrm{j}}, \mathrm{h}_{\mathrm{j}}$ e $\mathrm{d}_{\mathrm{j}}$ e estiver alocado em um bin $\mathrm{v}$ na posição $x_{j}, y_{j}$ e $z_{j}$, o centro de gravidade $\mathrm{G}$ do item $j$ no bin $\mathrm{v}$ é dado por: $\mathrm{G}_{\mathrm{x}}=x_{j}+w_{j} / 2$, $\mathrm{G}_{\mathrm{y}}=y_{j}+h_{j} / 2 \mathrm{e}_{\mathrm{z}}=z_{j}+d_{j} / 2$; sendo possível associar em $\mathrm{G}$ a força $\mathrm{P}_{\mathrm{j}}$ (peso do item).

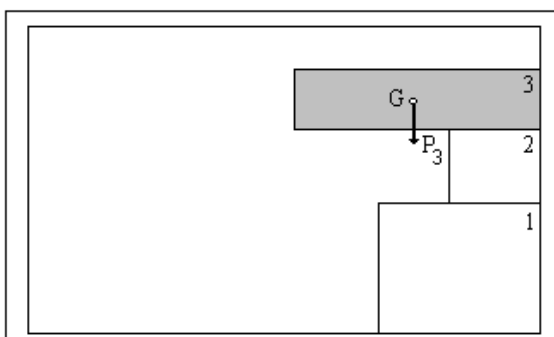

(a)

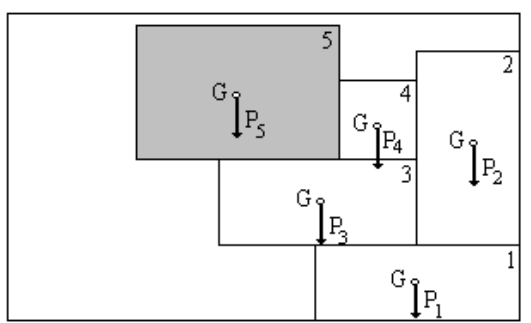

(b)

Figura 4 - Empacotamento visto por um observador na lateral do bin, aos itens 3 (4.a) e 5 (4.b), é feito o estudo da estabilidade estática (ou não) do conjunto resultante.

A Figura 4 ilustra as situações que podem gerar instabilidade estática nos itens já empacotados quando da inserção de um item adicional, no caso: item 3 (Figura 4a) e item 5 (Figura $4 \mathrm{~b}$ ). Também, foi suposto que os conjuntos de itens, já empacotados, encontravam-se em equilíbrio antes da inserção desses novos itens.

Na Figura (4.a) vê-se que o centro de gravidade G do item a ser empacotado, no caso item 3, não se encontra "apoiado" (linha de ação da força) sobre uma superfície em equilíbrio, provocando um desequilíbrio no mesmo. Entretanto, o item 3 estaria em equilíbrio caso G encontrasse apoiado: sobre o solo do bin; ou sobre a superfície rígida de algum outro item; ou ainda, indiretamente sobre as superfícies de alguns itens, conforme apresentado adiante. 
Na Figura (4.b) mostra que o centro de gravidade $\mathrm{G}$ do item a ser inserido, no caso item 5, está apoiado diretamente sobre a superfície do item 3, mas que há a possibilidade, daquele item, provocar instabilidade estática nas caixas previamente empacotadas. A inserção do item 5 na posição indicada pela Figura (4.b), implicará em um deslocamento dos itens 5, 3 e 4 , gerado pela rotação do item 3 . Note-se que isto decorre do fato do momento resultante dos itens empacotados ser diferente de zero em relação ao centro de gravidade resultante do empacotamento (Figura 8).

São apresentados, a seguir, a influência das forças e momentos no empacotamento. Um item estará em equilíbrio estático quando as forças externas a ele atuantes tiverem uma resultante nula e idem aos momentos. Desta forma, obtemos as seguintes condições necessárias e suficientes para o equilíbrio de um item, em relação a um dado ponto $\mathrm{O}$ :

$$
\vec{R}=\sum \vec{F}=\overrightarrow{0} \quad \overrightarrow{M_{O}^{R}}=\sum \overrightarrow{M_{O}}=\sum(\vec{r} \times \vec{F})=\overrightarrow{0}
$$

onde $\vec{F}$ e $\overrightarrow{\mathrm{r}}$ representam, respectivamente, a força e o vetor posição de aplicação da força $F$ no item, e $\vec{r} \times \vec{F}$ é o produto vetorial.

Decompondo cada força e cada momento em suas componentes retangulares $(x, y$ e $z)$, encontramos as condições necessárias e suficientes para o equilíbrio de um item,

$$
\begin{array}{lll}
\Sigma F_{x}=0 & \Sigma F_{y}=0 & \Sigma F_{z}=0 \\
\Sigma M_{x}=0 & \Sigma M_{y}=0 & \Sigma M_{z}=0
\end{array}
$$

As equações (1) expressam o fato de que as componentes das forças externas devem ser compensadas nas direções $x, y$ e $z$. As equações (2) expressam o fato de que os momentos das forças externas, em relação aos eixos $x, y$ e $z$, se anulam. O sistema das forças externas, por conseguinte, não irá gerar movimentos de translação ou de rotação no item considerado.

A Figura 5 mostra o momento, em relação ao ponto $\mathrm{O}$, de um corpo sobre a ação da intensidade de uma força atuando no ponto A.

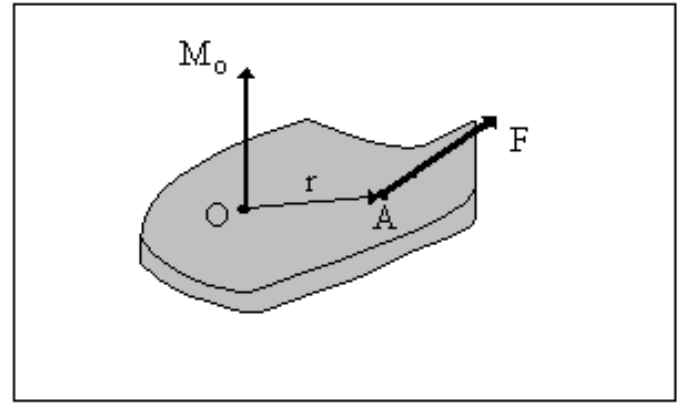

Figura 5 - Momento $\left(M_{\mathrm{O}}\right)$ de um corpo sobre a ação de uma força.

Se um corpo puder ser dividido em diversos outros, o seu centro de gravidade $G=(G x, G y, G z)$ pode ser determinado de acordo com as equações abaixo:

$$
\mathrm{Gx}=\Sigma(x . P) / \Sigma P \quad \mathrm{~Gy}=\Sigma(y \cdot P) / \Sigma P \quad \mathrm{Gz}=\Sigma(z \cdot P) / \Sigma P
$$


onde $x, y$ e $z$ são as coordenadas do centro de gravidade dos diversos corpos e $P$ é o módulo da força peso de cada um dos corpos.

Se o corpo é feito de material homogêneo, como adotado por hipótese para o 3D-BPP, o seu centro de gravidade coincide com o centróide de seu volume e podem ser utilizadas as seguintes equações:

$$
\mathrm{Gx}=\Sigma(x . V) / \Sigma V \quad \mathrm{~Gy}=\Sigma(y \cdot V) / \Sigma V \quad \mathrm{Gz}=\Sigma(z . V) / \Sigma V
$$

onde $V$ representa o volume de cada um dos corpos.

Analisando a situação ilustrada pela Figura (4.a) constata-se que, sendo $E$ o conjunto dos itens já alocados no referido bin, com estabilidade estática, a seguinte condição para que o item 3 fique em equilíbrio deve ser satisfeita:

$$
\begin{gathered}
\left(y_{3}=0\right) \\
\text { ou } \\
\left(\exists s \in E \text { tal que }\left(y_{3}=y_{s}+h_{s}\right),\left(x_{s} \leq \mathrm{Gx}_{3} \leq x_{s}+w_{s}\right) \text { e }\left(z_{s} \leq \mathrm{Gz}_{3} \leq z_{s}+d_{s}\right)\right)
\end{gathered}
$$

ou

(Existem $q$ e $r \in E ; y_{3}=y_{R}=y_{Q}, \mathrm{Gx}_{3}=\lambda x_{Q}+(1-\lambda) x_{R}$ e Gz$z_{3}=\lambda z_{Q}+(1-\lambda) z_{R}$,

$$
\text { para algum } 0 \leq \lambda \leq 1 \text { ) }
$$

onde $\mathrm{Q}=\left(x_{Q}, y_{Q}, z_{Q}\right)$ e $\mathrm{R}=\left(x_{R}, y_{R}, z_{R}\right)$, com $x_{q} \leq x_{Q} \leq x_{q}+w_{q}, y_{Q}=y_{q}+h_{q}, z_{q} \leq z_{Q} \leq z_{q}+d_{q}$, $x_{r} \leq x_{R} \leq x_{r}+w_{r}, y_{R}=y_{r}+h_{r}$ e $z_{r} \leq z_{R} \leq z_{r}+d_{r}$.

A condição (5) indica que o item está localizado no solo do bin. A condição (6), representada pela Figura 6, descreve o equilíbrio do item 3, quando o seu centro de gravidade G estiver apoiado diretamente na superfície de cima de outro item (s).

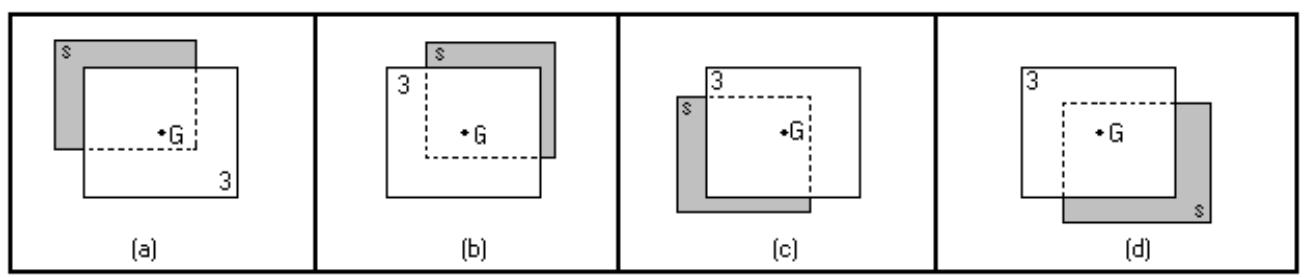

Figura 6 - Centro de gravidade $\mathrm{G}$ apoiado diretamente sobre a superfície de outra caixa, vista de topo.

A condição (7), representada pela Figura 7, especifica que para o item 3 estar em equilíbrio é necessário que exista um plano contendo o centro de gravidade $\mathrm{G}$, e os pontos $\mathrm{Q}$ e $\mathrm{R}$, onde $\mathrm{Q}$ e R estão nas regiões das superfícies de cima dos itens q e r, respectivamente, que tocam a superfície de baixo daquela caixa. Pelo menos uma das condições (5), (6) ou (7), deve ser satisfeita a fim de garantir a estabilidade estática de qualquer item que, porventura, venha a ser inserido dentro de um bin. 


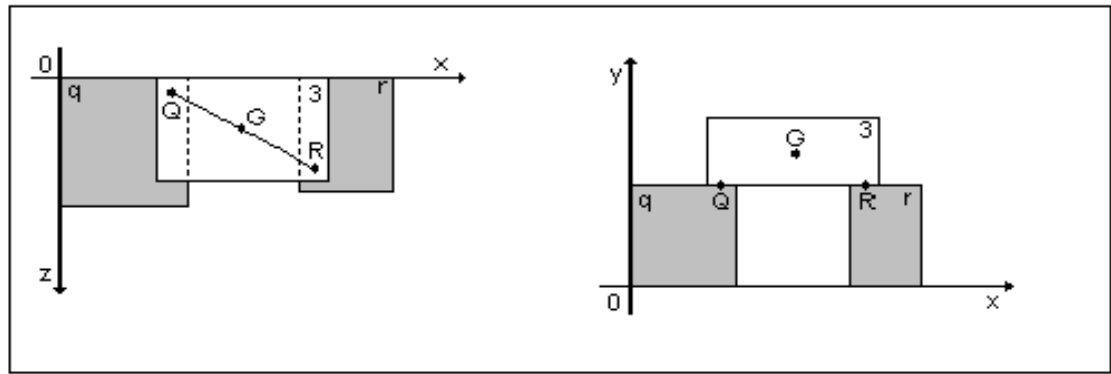

Figura 7 - Centro de gravidade $\mathrm{G}$ apoiado indiretamente sobre duas caixas, vistas de topo e de frente.

Também, foi analisada a situação ilustrada pela Figura (4.b) e verifica-se que para o empacotamento permanecer em equilíbrio estável é necessário que o momento resultante dos itens empacotados, em relação ao centro de gravidade do empacotamento, seja igual a zero. Note-se que a resultante das forças no empacotamento dos itens também será igual a zero. Resta, então, determinar o valor de $\overrightarrow{M_{O}^{R}}$ (momento resultante do sistema), onde $\mathrm{O}$ é o centro de gravidade do empacotamento dos itens, obtido pelas equações (4), $\vec{F}$ é a força peso de cada item no empacotamento e $\vec{r}$ é o vetor posição de $\mathrm{O}$ ao centro de gravidade $\mathrm{G}$ de cada item. Assim, se $\overrightarrow{M_{O}^{R}}=\overrightarrow{0}$ os itens do empacotamento estarão em equilíbrio, ou seja, a carga é estável. Caso contrário $\left(\overrightarrow{M_{O}^{R}} \neq \overrightarrow{0}\right)$, a carga está instável e não é possível inserir o item 5 naquela posição dentro do bin, pois ele irá gerar instabilidade no empacotamento. A Figura 8 , mostra o centro de gravidade do empacotamento ilustrado pela Figura (4.b).

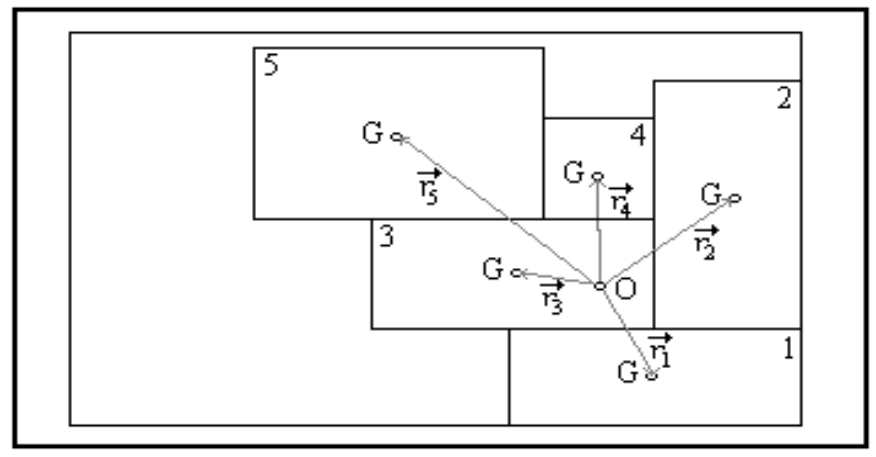

Figura 8 - Centro de gravidade do empacotamento (O) da Figura (4.b), vista lateral do bin.

A Heurística Com Estabilidade Estática (HCEE) segue a mesma estrutura de HSEE, com a diferença quando da inserção de um item. Mais especificamente, sempre que há a inserção de um item dentro de um bin com empacotamento estaticamente estável, prossegue-se da seguinte forma: primeiro é testado se uma das condições (5), (6) ou (7) é verdadeira, depois é verificado se $\overrightarrow{M_{O}^{R}}=\overrightarrow{0}$. Caso os resultados dessas operações forem verdadeiros então o 
referido item é alocado naquela posição dentro do referido contêiner, desta forma o sistema continuará em equilíbrio. Caso contrário, tenta-se alocar o referido item em outros pontos de cantos ou alocar outros itens no referido ponto de canto, e assim por diante, até que todos os itens estejam alocados com estabilidade. Note-se que a quantidade de operações necessárias para testar se um item pode ser incluído (ou não) em um ponto de canto mantendo a estabilidade estática, pode ser realizada em tempo constante, não alterando a ordem de convergência da heurística, que a exemplo de HSEE também é limitada, no tempo máximo de execução, por $O\left(n^{4}\right)$. Ainda, que em termos de espaço, as duas versões da heurística são claramente limitadas por $O(n)$.

\section{Experimentos Computacionais}

Um grande número de experimentos computacionais foi realizado, para avaliar o algoritmo proposto. Foram executadas as duas versões da heurística, HSEE e HCEE, comparando-os com a heurística $\mathrm{H}_{1}$, desenvolvida por Martello et al. (2000), a qual gera bons resultados práticos.

A heurística $\mathrm{H}_{1}$ aloca itens dentro de um bin de acordo com uma variação do preenchimento por camadas (prateleiras) sugerido em George \& Robinson (1980); sendo que os pontos de inserção são pontos de cantos bi-dimensionais. O problema resultante torna-se, então, o BPP o qual é resolvido pela política de First-Fit. A heurística $\mathrm{H}_{2}$ faz uma enumeração em árvore, onde os vértices são pontos de cantos tri-dimensionais. A geração da árvore é feita primeiramente em largura e a busca em profundidade é realizada para o preenchimento de um único bin. A heurística $\mathrm{H}_{2}$ foi implementada e testada, todavia, devido ao grande tempo de processamento e de memória utilizados - notadamente devido à grande quantidade de nós gerados pela árvore de busca - não foi possível a execução da mesma na máquina utilizada, para a quase totalidade dos testes realizados. Lembrando uma vez mais, que tanto $\mathrm{H}_{1}$ quanto $\mathrm{H}_{2}$ não levam em conta o equilíbrio da carga, quando do empacotamento dos itens.

As classes de testes são iguais àquelas definidas em Berkey \& Wang (1987) e Martello et al. (2000) e o conjunto de testes pode ser obtido por solicitação aos autores do presente artigo. As seguintes classes de problemas foram consideradas com uma quantidade de itens variando de 10 à 90 :

Para as classes de 1 à $5 W=H=D=100$; para todas as classes os dados foram gerados aleatoriamente a partir de funções de distribuição uniformes.

- Classe 1: A maioria dos itens são muitos altos e profundos,

$$
w_{j} \in[1,1 / 2 W], h_{j} \in[2 / 3 H, H] \text { e } d_{j} \in[2 / 3 D, D], j \in J \text {. }
$$

- Classe 2: A maioria dos itens são muitos largos e profundos,

$$
w_{j} \in[2 / 3 W, W], h_{j} \in[1,1 / 2 H] \text { e } d_{j} \in[2 / 3 D, D], j \in J .
$$

- Classe 3: A maioria dos itens são muitos altos e largos, $w_{j} \in[2 / 3 W, W], h_{j} \in[2 / 3 H, H]$ e $d_{j} \in[1,1 / 2 D], j \in J$.

- Classe 4: A maioria dos itens têm grandes dimensões, $w_{j} \in[1 / 2 W, W], h_{j} \in[1 / 2 H, H]$ e $d_{j} \in[1 / 2 D, D], j \in J$.

- Classe 5: A maioria dos itens têm pequenas dimensões, $w_{j} \in[1,1 / 2 W], h_{j} \in[1,1 / 2 H]$ e $d_{j} \in[1,1 / 2 D], j \in J$.

- Classe 6: problemas gerados aleatoriamente em um intervalo pequeno, $W=H=D=10, w_{j}, h_{j}, d_{j} \in[1,10]$. 
- Classe 7: problemas gerados aleatoriamente em um intervalo médio, $W=H=D=40, w_{j}, h_{j}, d_{j} \in[1,40]$.

- Classe 8: problemas gerados aleatoriamente em um intervalo grande, $W=H=D=100, w_{j}, h_{j}, d_{j} \in[1,100]$.

- Classe 9: problemas em que os itens são gerados (aleatoriamente) cuja solução é conhecida (puzzles), o empacotamento obedece a padrões não-guilhotinados, caso o problema fosse considerado de corte.

A amostra considerada para cada uma das classes tem tamanho 10 (para cada um dos valores de n). $\mathrm{H}_{1}$, HSEE e HCEE foram executados em um Pentium III (600 MHz com $128 \mathrm{Mb}$ de RAM) e o código foi implementado em Ansi C.

As Tabelas 1 e 2 apresentam o desvio médio em relação ao limitante inferior $\mathrm{L}_{2}$, conforme definido em Martello et al. (2000) e as médias dos tempos (segundos) de CPU. O desvio é definido como $\left(Z-\mathrm{L}_{2}\right) / \mathrm{L}_{2}$, onde $Z$ é o número mínimo de bins encontrado pelos algoritmos para empacotar todos os itens, em cada uma das instâncias. Já a Tabela 3 apresenta a média do volume do espaço não ocupado dos bins utilizados para o empacotamento dos itens.

Verifica-se da Tabela 1, que HSEE apresentou os melhores resultados em todas as classes, alcançando em média $66,5 \%, 66,8 \%, 75,7 \%, 67,9 \%, 62,6 \%, 62,6 \%, 55,7 \%, 62,3 \%$ e $61,9 \%$ dos resultados encontrados por $\mathrm{H}_{1}$, para as classes de 1 a 9 , respectivamente. Isto representa um índice médio de desvio de $64,6 \%$ dos resultados alcançados por HSEE em relação a $\mathrm{H}_{1}$, que é indicativo que HSEE produz soluções melhores que $\mathrm{H}_{1}$.

Tabela 1 - Desvio médio de $\mathrm{L}_{2}$ com respeito ao valor $\mathrm{Z}$ da solução encontrada.

\begin{tabular}{|c|c|c|c|c|c|c|c|c|c|c|c|c|c|c|}
\hline \multicolumn{2}{|c|}{ Classe } & 10 & 15 & 20 & 25 & 30 & 35 & 40 & 45 & 50 & 60 & 70 & 80 & 90 \\
\hline \multirow{3}{*}{1} & HCEE & 0,12 & 0,27 & 0,22 & 0,24 & 0,22 & 0,24 & 0,27 & 0,21 & 0,27 & 0,25 & 0,22 & 0,19 & 0,17 \\
\hline & HSEE & 0,08 & 0,27 & 0,22 & 0,20 & 0,19 & 0,20 & 0,25 & 0,19 & 0,25 & 0,23 & 0,21 & 0,18 & 0,16 \\
\hline & $\mathrm{H}_{1}$ & 0,24 & 0,33 & 0,32 & 0,30 & 0,31 & 0,32 & 0,37 & 0,30 & 0,33 & 0,36 & 0,30 & 0,25 & 0,22 \\
\hline \multirow{3}{*}{2} & HCEE & 0,42 & 0,70 & 0,53 & 0,77 & 0,80 & 0,70 & 0,73 & 0,65 & 0,67 & 0,69 & 0,69 & 0,61 & 0,56 \\
\hline & HSEE & 0,15 & 0,29 & 0,19 & 0,28 & 0,25 & 0,19 & 0,22 & 0,21 & 0,24 & 0,16 & 0,18 & 0,17 & 0,17 \\
\hline & $\mathrm{H}_{1}$ & 0,30 & 0,49 & 0,22 & 0,42 & 0,41 & 0,26 & 0,30 & 0,30 & 0,37 & 0,24 & 0,27 & 0,24 & 0,22 \\
\hline \multirow{3}{*}{3} & HCEE & 0,36 & 0,48 & 0,32 & 0,23 & 0,31 & 0,30 & 0,27 & 0,22 & 0,29 & 0,20 & 0,22 & 0,17 & 0,19 \\
\hline & HSEE & 0,36 & 0,40 & 0,32 & 0,22 & 0,27 & 0,25 & 0,25 & 0,20 & 0,25 & 0,17 & 0,21 & 0,17 & 0,17 \\
\hline & $\mathrm{H}_{1}$ & 0,41 & 0,53 & 0,39 & 0,28 & 0,35 & 0,35 & 0,33 & 0,29 & 0,35 & 0,27 & 0,28 & 0,23 & 0,22 \\
\hline \multirow{3}{*}{4} & HCEE & 0,03 & 0,03 & 0,01 & 0,02 & 0,04 & 0,03 & 0,03 & 0,03 & 0,04 & 0,02 & 0,04 & 0,03 & 0,03 \\
\hline & HSEE & 0,03 & 0,01 & 0,01 & 0,02 & 0,04 & 0,03 & 0,03 & 0,03 & 0,04 & 0,02 & 0,04 & 0,03 & 0,03 \\
\hline & $\mathrm{H}_{1}$ & 0,03 & 0,03 & 0,03 & 0,05 & 0,05 & 0,04 & 0,04 & 0,05 & 0,04 & 0,03 & 0,06 & 0,03 & 0,05 \\
\hline \multirow{3}{*}{5} & HCEE & 0,30 & 0,47 & 0,36 & 0,42 & 0,36 & 0,31 & 0,58 & 0,47 & 0,48 & 0,54 & 0,38 & 0,41 & 0,31 \\
\hline & HSEE & 0,30 & 0,42 & 0,30 & 0,40 & 0,30 & 0,22 & 0,43 & 0,44 & 0,37 & 0,41 & 0,32 & 0,31 & 0,25 \\
\hline & $\mathrm{H}_{1}$ & 0,45 & 0,71 & 0,51 & 0,56 & 0,47 & 0,44 & 0,68 & 0,67 & 0,65 & 0,67 & 0,47 & 0,48 & 0,37 \\
\hline \multirow{3}{*}{6} & HCEE & 0,28 & 0,29 & 0,17 & 0,18 & 0,24 & 0,25 & 0,19 & 0,20 & 0,25 & 0,20 & 0,22 & 0,19 & 0,16 \\
\hline & HSEE & 0,28 & 0,29 & 0,20 & 0,21 & 0,20 & 0,22 & 0,17 & 0,20 & 0,24 & 0,20 & 0,21 & 0,19 & 0,14 \\
\hline & $\mathrm{H}_{1}$ & 0,47 & 0,47 & 0,32 & 0,32 & 0,36 & 0,39 & 0,30 & 0,31 & 0,37 & 0,30 & 0,31 & 0,25 & 0,22 \\
\hline \multirow{3}{*}{7} & HCEE & 0,63 & 0,46 & 0,38 & 0,50 & 0,70 & 0,58 & 0,52 & 0,63 & 0,54 & 0,61 & 0,72 & 0,59 & 0,54 \\
\hline & HSEE & 0,43 & 0,29 & 0,27 & 0,31 & 0,47 & 0,44 & 0,44 & 0,48 & 0,41 & 0,47 & 0,54 & 0,37 & 0,32 \\
\hline & $\mathrm{H}_{1}$ & 0,83 & 0,61 & 0,62 & 0,69 & 1,07 & 0,78 & 0,81 & 0,82 & 0,60 & 0,71 & 0,79 & 0,55 & 0,52 \\
\hline \multirow{3}{*}{8} & HCEE & 0,50 & 0,40 & 0,26 & 0,41 & 0,51 & 0,42 & 0,65 & 0,55 & 0,47 & 0,32 & 0,42 & 0,42 & 0,49 \\
\hline & HSEE & 0,40 & 0,25 & 0,21 & 0,27 & 0,32 & 0,30 & 0,42 & 0,42 & 0,38 & 0,23 & 0,32 & 0,30 & 0,36 \\
\hline & $\mathrm{H}_{1}$ & 0,68 & 0,55 & 0,33 & 0,58 & 0,58 & 0,44 & 0,70 & 0,60 & 0,57 & 0,38 & 0,40 & 0,42 & 0,47 \\
\hline \multirow{3}{*}{9} & HCEE & 0,08 & 0,50 & 0,62 & 1,22 & 1,02 & 1,45 & 1,35 & 1,45 & 1,58 & 3,12 & 1,85 & 2,32 & 3,70 \\
\hline & HSEE & 0,05 & 0,47 & 0,40 & 0,68 & 0,40 & 0,92 & 0,73 & 0,77 & 1,00 & 1,77 & 0,92 & 1,02 & 1,67 \\
\hline & $\mathrm{H}_{1}$ & 0,23 & 0,67 & 0,50 & 1,07 & 0,82 & 1,42 & 1,25 & 1,18 & 1,60 & 2,40 & 1,70 & 1,90 & 2,70 \\
\hline
\end{tabular}


Comparando HCEE com $\mathrm{H}_{1}$, levando em conta a Tabela 1, verifica-se que HCEE apresentou melhores resultados que $\mathrm{H}_{1}$ em sete das nove classes, alcançando as seguintes médias para cada um dos conjuntos de testes: $73,1 \%, 210,8 \%, 83,1 \%, 71,6 \%, 75,5 \%, 64,2 \%, 78,7 \%$, $86,8 \%$ e $116,1 \%$ daqueles encontrados por $\mathrm{H}_{1}$, para as classes de 1 a 9 , respectivamente. Em termos médios de quantidade de bins utilizados, $\mathrm{HCEE} \mathrm{e} \mathrm{H}_{1}$ podem ser consideradas quase equivalentes, i.e. tem-se $4,5 \%$ menos bins abertos por HCEE que $\mathrm{H}_{1}$. Todavia, HCEE teria um índice médio de desvio de $76,1 \%$ menor que $\mathrm{H}_{1}$, se não fossem computados as médias das classes $2(210,8 \%)$ e $9(116,1 \%)$. Nessas duas classes HCEE obteve os piores resultados, quando comparado a $\mathrm{H}_{1}$. Para a Classe 9, uma provável explicação do por quê HCEE produz piores resultados vem do fato dos itens terem de ser empacotados de forma "não-guilhotinada", e a colocação de uma peça "no meio" de um conjunto de itens já empacotados, via de regra, não ocorre, devido aos encaixes estarem restritos aos pontos de cantos, por exemplo, como na figura a seguir.

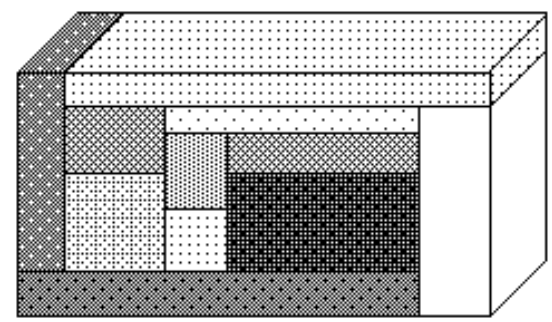

Figura 9 - Empacotamento "não-guilhotinado".

É importante notar ainda, que configurações como a da Figura 10, apresentada a seguir, embora fisicamente estáveis não podem ser geradas por HCEE. Supondo que a ordem de empacotamento dos itens, seja: item 1, item 4 , item 2 e item 3 . O item 2 não poderia ser inserido no bin em questão, pois o centro de gravidade do mesmo não está sobre o item $1 \mathrm{e}$ também por que a altura dos itens 1 e 4 não é igual $(\mathrm{h} 1>\mathrm{h} 4)$.

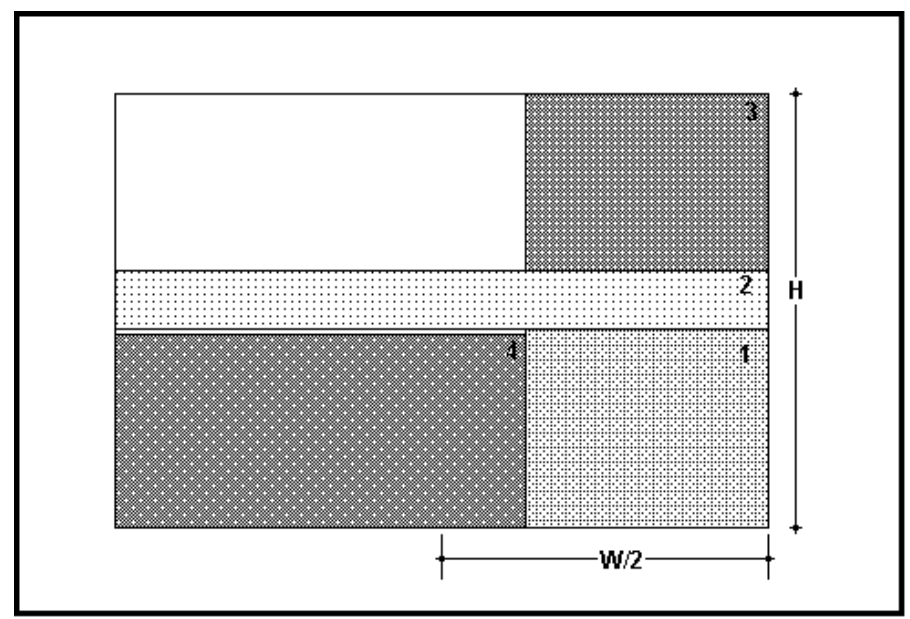

Figura 10 - Empacotamento estável que HCEE não pode gerar. 
HCEE gerou em média 9,8\%, 215,5\%, 9,8\%, 5,5\%, 20,5\%, 2,5\%, 41,2\%, 39,2\% e 87,5\% a mais do que os resultados encontrados por HSEE para as classes de 1 a 9 , respectivamente. Isto dá uma idéia da quantidade de bins usados a mais de uma solução sem estabilidade para uma solução que leva em conta os critérios de estabilidade estática. Com base nesses valores, HCEE gerou uma média global de 47,9\% mais bins que HSEE. Se as classes 2 e 9 fossem retiradas deste cálculo o aumento cairia para $18,3 \%$.

Na Figura 11 a seguir, apresenta-se uma solução gerada por $\mathrm{H}_{1}$, onde fica clara a necessidade da inserção de prateleiras, caso contrário os conjuntos são claramente instáveis. Note-se que nos bins 3, 4 e 6 o empacotamento é estável. Alguns itens (nos bins 1, 2 e 5) não foram apresentados para uma melhor visualização do empacotamento instável.

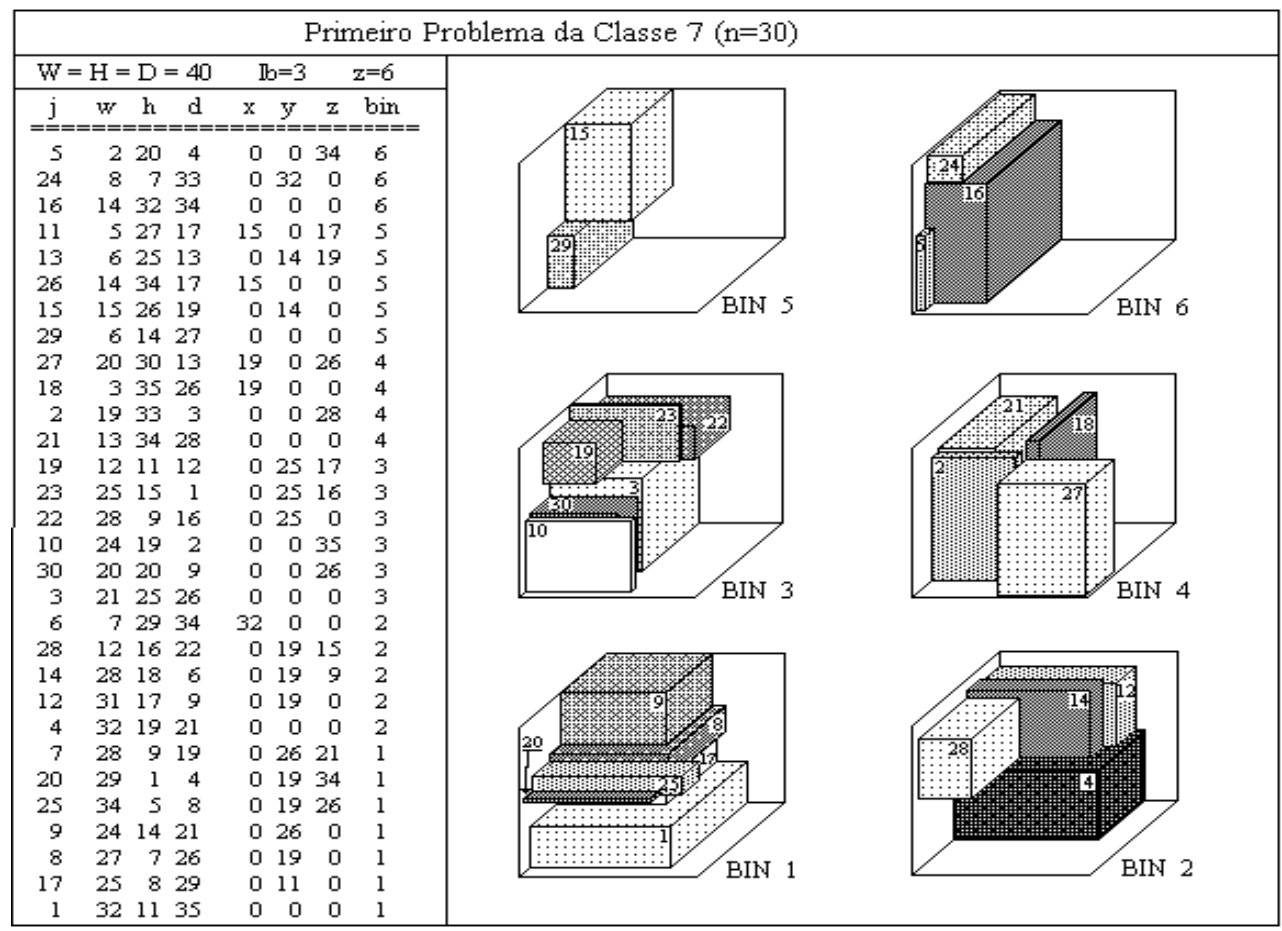

Figura 11 - Exemplo de uma solução apresentada por $\mathrm{H}_{1}$ sem estabilidade estática.

Na Figura 12 tem-se a representação gráfica do empacotamento do mesmo conjunto de itens quando da utilização de HCEE. Apenas o item 2 não está apresentado, por encontrar-se sobre a superfície do solo do bin 1 e atrás do item 10 , no bin 1 . 


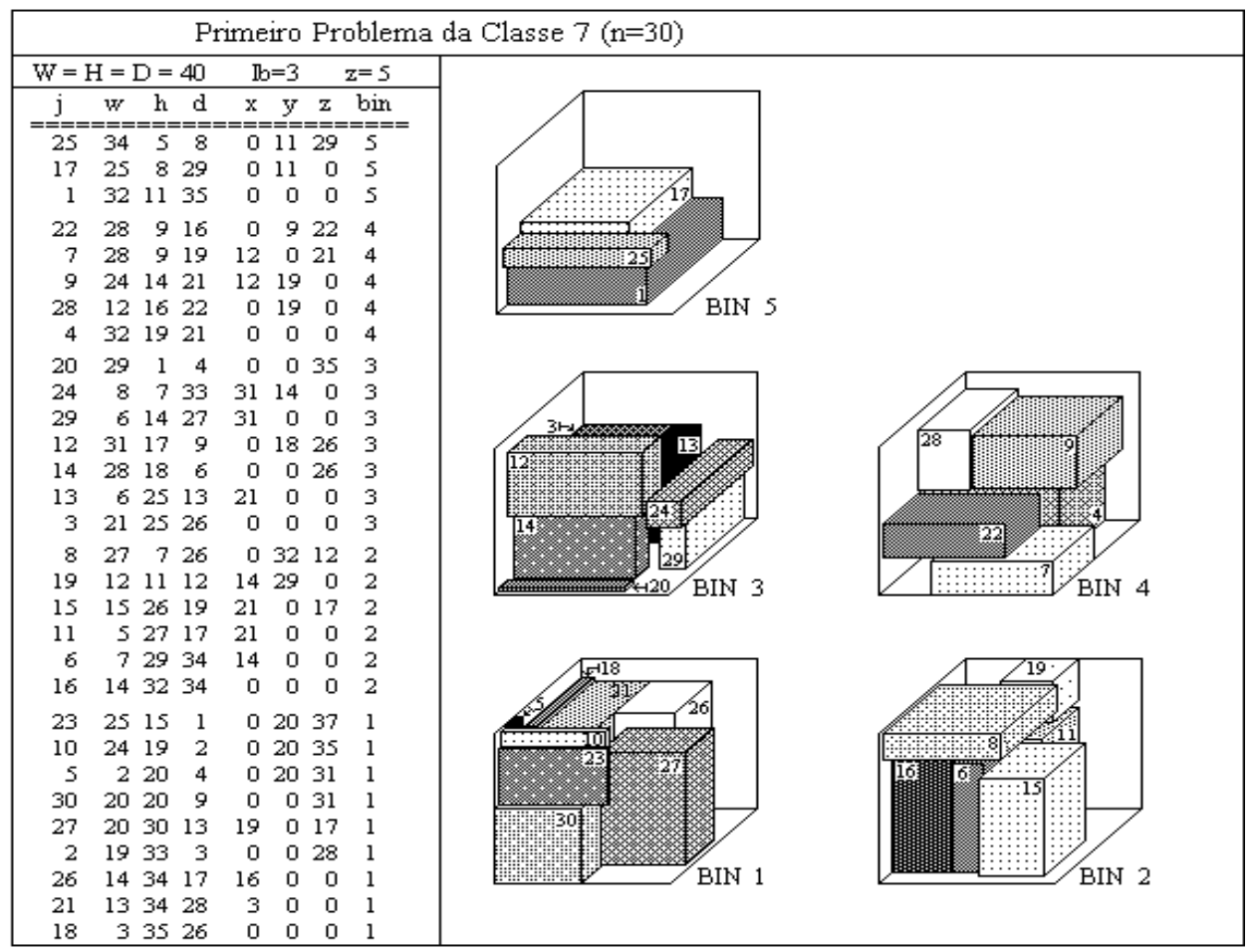

Figura 12 - Exemplo de uma solução apresentada por HCEE.

As tabelas apresentadas a seguir têm a comparação do tempo de processamento e a média do volume do espaço não ocupado dos bins de $\mathrm{H}_{1}$, HSEE e HCEE.

Tabela 2 - Tempo médio expresso em segundos de CPU para encontrar a solução pelas heurísticas.

\begin{tabular}{|c|c|c|c|c|c|c|c|c|c|c|c|c|c|c|}
\hline \multicolumn{2}{|c|}{ Classe } & 10 & 15 & 20 & 25 & 30 & 35 & 40 & 45 & 50 & 60 & 70 & 80 & 90 \\
\hline \multirow{3}{*}{1} & HCEE & 0,00 & 0,01 & 0,01 & 0,01 & 0,01 & 0,01 & 0,01 & 0,02 & 0,02 & 0,02 & 0,03 & 0,04 & 0,04 \\
\hline & HSEE & 0,00 & 0,00 & 0,01 & 0,01 & 0,01 & 0,01 & 0,01 & 0,01 & 0,02 & 0,02 & 0,03 & 0,03 & 0,04 \\
\hline & $\mathrm{H}_{1}$ & 0,01 & 0,00 & 0,00 & 0,00 & 0,00 & 0,02 & 0,05 & 0,08 & 0,09 & 0,19 & 0,26 & 0,24 & 0,30 \\
\hline \multirow{3}{*}{2} & HCEE & 0,00 & 0,01 & 0,01 & 0,01 & 0,01 & 0,01 & 0,02 & 0,02 & 0,02 & 0,03 & 0,04 & 0,05 & 0,06 \\
\hline & HSEE & 0,00 & 0,01 & 0,00 & 0,01 & 0,01 & 0,01 & 0,02 & 0,02 & 0,02 & 0,02 & 0,03 & 0,04 & 0,04 \\
\hline & $\mathrm{H}_{1}$ & 0,00 & 0,00 & 0,00 & 0,01 & 0,01 & 0,04 & 0,05 & 0,10 & 0,13 & 0,23 & 0,23 & 0,27 & 0,30 \\
\hline \multirow{3}{*}{3} & HCEE & 0,00 & 0,01 & 0,01 & 0,01 & 0,01 & 0,01 & 0,02 & 0,02 & 0,02 & 0,03 & 0,03 & 0,04 & 0,04 \\
\hline & HSEE & 0,00 & 0,00 & 0,01 & 0,01 & 0,01 & 0,01 & 0,01 & 0,02 & 0,02 & 0,02 & 0,03 & 0,04 & 0,04 \\
\hline & $\mathrm{H}_{1}$ & 0,00 & 0,00 & 0,00 & 0,00 & 0,01 & 0,02 & 0,08 & 0,08 & 0,12 & 0,21 & 0,25 & 0,27 & 0,30 \\
\hline \multirow{3}{*}{4} & HCEE & 0,00 & 0,00 & 0,00 & 0,01 & 0,00 & 0,01 & 0,01 & 0,01 & 0,01 & 0,01 & 0,02 & 0,03 & 0,04 \\
\hline & HSEE & 0,00 & 0,00 & 0,00 & 0,01 & 0,00 & 0,01 & 0,01 & 0,01 & 0,01 & 0,01 & 0,02 & 0,03 & 0,04 \\
\hline & $\mathrm{H}_{1}$ & 0,00 & 0,00 & 0,00 & 0,00 & 0,00 & 0,00 & 0,00 & 0,01 & 0,00 & 0,01 & 0,01 & 0,01 & 0,01 \\
\hline \multirow{3}{*}{5} & HCEE & 0,01 & 0,01 & 0,01 & 0,01 & 0,01 & 0,02 & 0,03 & 0,02 & 0,03 & 0,04 & 0,05 & 0,06 & 0,07 \\
\hline & HSEE & 0,01 & 0,01 & 0,01 & 0,01 & 0,02 & 0,02 & 0,02 & 0,02 & 0,02 & 0,04 & 0,05 & 0,06 & 0,07 \\
\hline & $\mathrm{H}_{1}$ & 0,00 & 0,00 & 0,01 & 0,00 & 0,01 & 0,01 & 0,01 & 0,01 & 0,01 & 0,01 & 0,01 & 0,02 & 0,02 \\
\hline \multirow{3}{*}{6} & HCEE & 0,00 & 0,01 & 0,01 & 0,01 & 0,01 & 0,01 & 0,01 & 0,01 & 0,02 & 0,02 & 0,02 & 0,03 & 0,03 \\
\hline & HSEE & 0,00 & 0,00 & 0,01 & 0,01 & 0,01 & 0,01 & 0,01 & 0,01 & 0,02 & 0,02 & 0,02 & 0,03 & 0,03 \\
\hline & $\mathrm{H}_{1}$ & 0,00 & 0,01 & 0,00 & 0,01 & 0,00 & 0,00 & 0,00 & 0,00 & 0,00 & 0,00 & 0,01 & 0,01 & 0,03 \\
\hline
\end{tabular}


Tabela 2A - Tempo médio expresso em segundos de CPU para encontrar a solução pelas heurísticas (continuação).

\begin{tabular}{|c|c|c|c|c|c|c|c|c|c|c|c|c|c|c|}
\hline \multicolumn{2}{|c|}{ Classe } & 10 & 15 & 20 & 25 & 30 & 35 & 40 & 45 & 50 & 60 & 70 & 80 & 90 \\
\hline \multirow{3}{*}{7} & HCEE & 0,01 & 0,01 & 0,01 & 0,01 & 0,02 & 0,01 & 0,02 & 0,02 & 0,03 & 0,03 & 0,03 & 0,05 & 0,05 \\
\hline & HSEE & 0,01 & 0,01 & 0,01 & 0,01 & 0,01 & 0,01 & 0,02 & 0,02 & 0,03 & 0,02 & 0,03 & 0,04 & 0,05 \\
\hline & $\mathrm{H}_{1}$ & 0,00 & 0,00 & 0,00 & 0,00 & 0,00 & 0,01 & 0,01 & 0,01 & 0,01 & 0,01 & 0,01 & 0,01 & 0,01 \\
\hline \multirow{3}{*}{8} & HCEE & 0,01 & 0,01 & 0,01 & 0,01 & 0,01 & 0,02 & 0,02 & 0,02 & 0,03 & 0,03 & 0,04 & 0,05 & 0,05 \\
\hline & HSEE & 0,01 & 0,01 & 0,01 & 0,01 & 0,01 & 0,01 & 0,02 & 0,02 & 0,03 & 0,02 & 0,03 & 0,04 & 0,05 \\
\hline & $\mathrm{H}_{1}$ & 0,00 & 0,00 & 0,00 & 0,00 & 0,01 & 0,00 & 0,00 & 0,00 & 0,00 & 0,01 & 0,01 & 0,02 & 0,03 \\
\hline \multirow{3}{*}{9} & HCEE & 0,00 & 0,01 & 0,01 & 0,01 & 0,01 & 0,02 & 0,02 & 0,02 & 0,03 & 0,03 & 0,05 & 0,07 & 0,08 \\
\hline & HSEE & 0,00 & 0,00 & 0,00 & 0,01 & 0,00 & 0,01 & 0,02 & 0,02 & 0,02 & 0,02 & 0,05 & 0,06 & 0,07 \\
\hline & $\mathrm{H}_{1}$ & 0,00 & 0,00 & 0,00 & 0,00 & 0,00 & 0,00 & 0,01 & 0,01 & 0,00 & 0,00 & 0,01 & 0,01 & 0,01 \\
\hline
\end{tabular}

Tabela 3 - Média do volume do espaço não ocupado dos bins.

\begin{tabular}{|c|c|c|c|c|c|c|c|c|c|c|c|c|c|c|}
\hline Classe & 10 & 15 & 20 & 25 & 30 & 35 & 40 & 45 & 50 & 60 & 70 & 80 & 90 & Média \\
\hline HCEE & 0,43 & 0,44 & 0,39 & 0,40 & 0,39 & 0,38 & 0,36 & 0,36 & 0,37 & 0,34 & 0,34 & 0,33 & 0,33 & 0,3738 \\
\hline 1 HSEE & 0,41 & 0,44 & 0,39 & 0,38 & 0,37 & 0,36 & 0,35 & 0,35 & 0,36 & ד נז, & 0,33 & 0,32 & 0,32 & ,3623 \\
\hline $\mathrm{H}_{1}$ & 0,48 & 0,46 & 0,44 & 0,43 & 0,43 & 0,41 & 0,41 & 0,40 & 0,40 & 0,39 & 0,38 & 0,36 & 0,35 &, 4108 \\
\hline HCEE & 0,55 & 0,56 & 0,55 & 0,56 & 0,56 & 0,55 & 0,54 & 0,52 & 0,51 & 0,55 & 0,52 & 0,51 & 0,49 & 0,5362 \\
\hline HSEE & 0,45 & 0,43 & 0,43 & 0,39 & 0,37 & 0,36 & 0,35 & 0,35 & 0,35 & 0,34 & 0,32 & & 0,32 & 3685 \\
\hline $\mathrm{H}_{1}$ & 0,50 & 0,51 & 0,44 & 0,45 & 0,43 & 0,40 & 0,39 & 0,39 & 0,41 & 0,39 & 0,37 & 0,37 & 0,35 & 0,4154 \\
\hline HCEE & 0,49 & 0,47 & & 0,40 & 0,39 & 0,3 & 0,38 & 0,35 & 0,36 & & 0,34 & & 0,33 & 0,3838 \\
\hline HSEE & 0,49 & 0,45 & 0,4 & 0,40 & 0,37 & 0,37 & 0,37 & & 0,34 & 33 & 0,33 & 33 & 32 & ,3738 \\
\hline $\mathrm{H}_{1}$ & 0,50 & 0,48 & 0,45 & 0,42 & 0,41 & 0,40 & 0,40 & 0,39 & 0,38 & 0,39 & 0,37 & 0,36 & 0,35 & 0,4077 \\
\hline HCEE & 0,52 & 0,49 & & 0,48 & 0,47 & 0,49 & 0,49 & 0,50 & 0,48 & 0,50 & 0,49 & 0,49 & 0,49 & 0,4915 \\
\hline HSEE & 0,52 & 0,48 & 0,50 & 0,48 & 0,47 & 0,49 & 0,49 & 0,50 & 0,48 & 0,50 & 0,49 & 0,49 & 0,49 & 0,4908 \\
\hline $\mathrm{H}_{1}$ & 0,52 & 0,49 & 0,51 & 0,49 & 0,48 & 0,49 & 0,50 & 0,50 & 0,48 & 0,50 & 0,50 & 0,49 & 0,50 & 0,4962 \\
\hline HCEE & 0,62 & 0,51 & & 0,48 & 0,43 & 0,42 & 0,44 & 0,39 & 0,39 & 0,40 & 0,37 & 0,37 & 0,35 & 0,4362 \\
\hline HSEE & 0,62 & 0,50 & 47 & 0,47 & 0,40 & 0,37 & 38 & 38 & 0,35 & 0,36 & 0,34 & 32 & 0,32 & 0,4062 \\
\hline $\mathrm{H}_{1}$ & 0,64 & 0,57 & 0,54 & 0,52 & 0,47 & 0,46 & 0,46 & 0,46 & 0,45 & 0,45 & 0,40 & 0,40 & 0,38 & 0,4769 \\
\hline HCEE & 0,46 & 0,41 & 38 & 0,34 & 0,31 & 0,31 & 0,31 & 0,28 & 0,27 & 0,26 & 0,23 & 0,25 & 0,23 & 0,3092 \\
\hline HSEE & & 0,41 & & 0,34 & 0,30 & 0,2 & 0,30 & 0,28 & 0,27 & 0,26 & 0,23 & 0,2 & 0,22 & 0,3062 \\
\hline $\mathrm{H}_{1}$ & 0,52 & 0,48 & 0,43 & 0,40 & 0,38 & 0,38 & 0,37 & 0,34 & 0,34 & 0,31 & 0,29 & 0,28 & 0,27 & 0,3685 \\
\hline HCEE & 0,63 & 0,58 & 0,52 & 0,52 & 0,51 & 0,47 & 0,46 & 0,47 & 0,46 & 0,45 & 0,44 & 0,44 & 0,43 & 0,4908 \\
\hline 7 HSEE & 0,61 & 0,51 & 0,48 & 0,46 & 0,42 & 0,42 & 0,43 & 0,42 & 0,41 & 0,40 & 0,37 & 0,3 & 0,33 & 0,4315 \\
\hline $\mathrm{H}_{1}$ & 0,69 & 0,61 & 0,59 & 0,58 & 0,59 & 0,53 & 0,54 & 0,52 & 0,48 & 0,48 & 0,46 & 0,43 & 0,42 & 0,5323 \\
\hline HCEE & & & & 0,48 & & & & & & & & & & 0,4385 \\
\hline 8 HSEE & 0,53 & 0,44 & 0,46 & 0,43 & 0,40 & 0,40 & 0,37 & 0,38 & 0,35 & 0,36 & 0,35 & 0,32 & 0,33 & 0,3938 \\
\hline $\mathrm{H}_{1}$ & 0,59 & 0,54 & 0,50 & 0,53 & 0,49 & 0,46 & 0,46 & 0,44 & 0,42 & 0,42 & 0,39 & 0,38 & 0,37 & 0,4608 \\
\hline HCEE & & 0,27 & & 0,45 & 0,47 & & 0,49 & & 0,50 & 0,56 & 0,57 & 0,59 & 0,66 & 0,4523 \\
\hline 9 HSEE & & 0,25 & & 0,28 & & & & & 0,37 & 0,37 & 0,40 & & 0,40 & 0,3000 \\
\hline $\mathrm{H}_{1}$ & 0,12 & 0,34 & 0,26 & 0,42 & 0,42 & 0,48 & 0,47 & 0,45 & 0,51 & 0,48 & 0,57 & 0,56 & 0,56 & 0,4338 \\
\hline
\end{tabular}

Ao se analisar a Tabela 2, constata-se que HCEE, HSEE e $\mathrm{H}_{1}$ são bastante rápidas. HCEE consumiu mais tempo que HSEE, devido ao tempo que se gasta para encontrar uma outra solução com estabilidade estática. Mesmo assim, os tempos obtidos por $\mathrm{H}_{1}$ e as duas versões da heurística podem ser considerados aceitáveis, pelo menos sob o ponto de vista prático/ industrial.

Verifica-se na Tabela 3, com base na coluna Média, que HCEE e $\mathrm{H}_{1}$ não conseguiram preencher, na média, nem mesmo $50 \%$ do volume dos bins utilizados para empacotar os itens, nas classes 2 e 7, respectivamente. Nas outras classes a média de ocupação dos bins foi 
superior a $50 \%$, tanto para $\mathrm{H}_{1}$ quanto para HCEE. É interessante notar que com a métrica utilizada para a composição desta tabela, HSEE não deve ser preterida à HCEE, pois para a Classe 9 e $\mathrm{n}=10$, o problema foi resolvido de forma exata para todos os casos. Na mesma classe 9, HSEE teve a melhor média de ocupação, chegando a preencher todos os bins em mais de $60 \%$ na média. Exceto nas classe 2 e 9, HCEE também teve um desempenho superior a $\mathrm{H}_{1}$.

\section{Conclusões}

Através dos experimentos computacionais, baseados em problemas da literatura, há a indicação que a versão em que a estabilidade estática não é considerada (HSEE) boas soluções são geradas e com tempo compatível com aquela sugerida em Martello et al. (2000). Foram introduzidos critérios para que o empacotamento dos itens nos contêineres formasse um conjunto estaticamente estável, o que no melhor do conhecimento dos autores é inédito. Mais ainda, que quando da incorporação desses à heurística proposta, o tempo de processamento continuou sendo adequado para aplicações industriais.

Estudos preliminares foram realizados para tratar também da estabilidade dinâmica do empacotamento. Todavia, devido à grande quantidade de fatores a serem considerados, e.g. coeficiente de atrito das caixas, velocidade e aceleração dos bins, e etc, não foi possível a padronização desses em um único estudo. Faz-se notar que em termos práticos, caso os bins não sofram variações abruptas de velocidade, inclinação e curvatura, o que ocorre por exemplo no transporte marítimo (contêineres em navios), o problema da estabilidade da carga no 3D-BPP, resolvido por HCEE, pode ser considerado como melhor alternativa àquelas sugeridas na literatura.

Um pacote gráfico foi também gerado, onde é possível acompanhar como se dá o preenchimento dos bins, item a item. Por exemplo, as Figuras 2, 11 e 12 foram por ele gerada. Os códigos fontes da heurística proposta, versões com e sem estabilidade, e do pacote gráfico podem ser obtidos por solicitação junto aos autores do presente artigo.

\section{Agradecimentos}

Os autores agradecem apoio financeiro da Fapesp, CNPq e Capes. Agradecem também aos Profs. Silvano Martello da Universidade de Bolonha e David Pisinger da Universidade de Copenhagem por haverem cedido o código fonte em $\mathrm{C}$ e discussões sobre a implementação das heurísticas por eles propostas.

\section{Referências Bibliográficas}

(1) Beer, F.P. \& Johnston, E.R. (1976). Mechanics for engineers: statics and dynamics. McGraw Hill, New York.

(2) Berkey, J.O. \& Wang, P.Y. (1987). Two dimensional finite bin packing algorithms. Journal of the Operational Research Society, 38, 423-429.

(3) Chen, C.S.; Lee, S.M. \& Shen, Q.S. (1995). An analytical model for the container loading problem. European Journal of Operational Research, 80, 68-76. 
(4) Corcoran, A.L. \& Wainwright, R.L. (1992). A genetic algorithm for packing in three dimensions. Proceedings of the 1992 ACM/SIGAPP Symposium on Applied Computing, 1021-1030.

(5) Cormen, T.H.; Leiserson, C.E \& Rivest, R.L. (1990). Introduction to Algorithms. MIT Press, Cambridge.

(6) Dowsland, W.B. (1991). Three-dimensional packing solution approaches and heuristics development. International Journal of Production Research, 29(8), 1673-1685.

(7) Garey, M.R. \& Johnson, D.S. (1979). Computers and Intractability: A guide to the Theory of NP-Completeness. W.H. Freeman, San Francisco.

(8) George, J.A. \& Robinson, D.F. (1980). A heuristic for packing boxes into a container. Computers and Operational Research, 7, 147-156.

(9) Martello, S. \& Vigo, D. (1998). Exact solution of the two-dimensional finite bin packing problem. Management Science, 44, 388-399.

(10) Martello, S.; Pisinger, D. \& Vigo, D. (2000). The three-dimensional bin packing problem. Operations Research, 48, 256-267.

(11) Meriam, J.L. \& Kraige, L.G. (1986). Engineering Mechanics: Dynamics (vol. 2). Wiley, New York.

(12) Pisinger, D.; Farøe, O. \& Zachariasen, M. (1999). Guided Local Search for the threedimensional bin packing problem. Techinical Report, DIKU 99-13, Københavns Universitet, Datalogisk institut.

(13) Scheithauer, G. (1991). A three-dimensional bin packing algorithm. J. Inform. Process. Cybernet., 27, 263-271.

(14) Soma, N.Y.; Yanasse, H.H. \& Maculan, N. (1999). A heuristic for the bin packing problem. Relatório Técnico, CTA/ITA-IEC/AE-001/99, Instituto Tecnológico de Aeronáutica, resumo apresentado no IFORS 1999, Beijing, China. 